\title{
SELEÇÃO SIMULTÂNEA PARA PORTE REDUZIDO E ALTA PRODUÇÃO DE LÁTEX EM SERINGUEIRA $\left({ }^{1}\right)$
}

\author{
REGINALDO BRITO DA COSTA $\left(2^{*}\right)$; MARCOS DEON VILELA DE RESENDE $\left({ }^{3}\right)$; \\ PAULO DE SOUZA GONÇALVES $\left({ }^{4}\right)$; LINCOLN CARLOS SILVA DE OLIVEIRA $\left({ }^{2}\right)$; \\ LUÍS CARLOS VINHAS ÍTAVO $\left({ }^{2}\right)$; RAUL ALFFONSO RODRIGUES ROA $\left({ }^{2,5}\right)$
}

\begin{abstract}
RESUMO
Os objetivos deste trabalho foram avaliar a variabilidade genética e a possibilidade de sucesso na seleção simultânea para porte baixo e alta produção de látex considerando as variáveis relacionais: produtividade de látex por unidade de altura (PBA) e produtividade de látex por área basal das plantas (PBB) como novos caracteres no melhoramento da seringueira (Hevea brasiliensis). A seleção baseada nas referidas variáveis permitem a identificação de novos materiais genéticos destinados a plantios adensados. Vinte e duas progênies de meios-irmãos foram estabelecidas no município de Votuporanga (SP), sob delineamento de blocos ao acaso com 22 tratamentos, cinco repetições e dez plantas por parcela, no espaçamento de $3 \times 2 \mathrm{~m}$ em linhas simples. Aos três anos de idade, as progênies foram avaliadas quanto aos caracteres em estudo. A combinação dos dois caracteres aumentou a variabilidade genética disponível para seleção sendo possível desenvolver eficiente melhoramento genético simultâneo para o aumento da produção de borracha e redução do porte da planta. Portanto, é possível melhorar os dois caracteres (PB e PBB) simultaneamente, não havendo prejuízo no caráter principal (PB). As altas correlações genéticas entre PBB e PBA $(0,842)$ e entre PBB e altura $(-0,578)$ revelam que a seleção baseada em PBB resultará em redução da altura. O tipo de seleção foi eficiente em aumentar a produção de borracha, reduzindo substancialmente a altura e pouco o perímetro do caule. Esses efeitos são desejáveis em um programa de melhoramento da seringueira visando à seleção de genótipos para plantios adensados.
\end{abstract}

Palavras-chave: Hevea brasiliensis, melhoramento genético, produção de borracha, porte reduzido.

\section{ABSTRACT \\ SIMULTANEOUS SELECTION FOR SHORT PLANT HEIGHT AND HIGH RUBBER YIELD IN RUBBER TREE}

Goals of this work were to evaluate genetic variability and potential of successful simultaneous selection for short stature and high rubber yield, considering relational variables: rubber yield per height unit (PBA) and rubber yield per plant basal area (PBB), both as new traits in rubber tree genetic improvement. Selection based on these variables allows identification of new genetic material suitable for high density plantations. Twenty-two half-sib progenies of half-sibs were established in Votuporanga. The trial was carried out with 22 treatments with five replications each and 10 plants per plot, planted in single lines at density of $3 \times 2 \mathrm{~m}$. Plots were randomly distributed. At three year old age, progenies were evaluated and showed that a combination of those two traits increased genetic variability available

$\left({ }^{1}\right)$ Recebido para publicação em 2 de julho de 2007 e aceito em 8 de fevereiro de 2008.

$\left({ }^{2}\right)$ Universidade Católica Dom Bosco (UCDB), Programa de Mestrado em Desenvolvimento Local, Av. Tamandaré, 6000, Jardim Seminário, Caixa Postal 100, 79117-100 Campo Grande (MS). E-mail: reg.brito.costa@gmail.com $\left(^{*}\right)$ Autor correspondente; lincoln@ucdb.br; itavo@ucdb.br

$\left({ }^{3}\right)$ Embrapa - Centro Nacional de Pesquisa de Florestas (CNPF), Caixa Postal 31, 83411-000 Colombo (PR). E-mail: deon@cnpf.embrapa.br.

$\left({ }^{4}\right)$ Programa Seringueira - Instituto Agronômico de Campinas (IAC), Caixa Postal 28, 13001-970 Campinas, (SP)

$\left({ }^{5}\right)$ Bolsista CAPES. E-mail: roabio@gmail.com. 
for selection. Results also demonstrated that it is possible to have an efficient genetic improvement for simultaneously increasing rubber yield while reducing plant height. Therefore, it is possible to improve these two traits (PB and $\mathrm{PBB}$ ) simultaneously, without losses on the main traits (PB). High correlations between PBB and PBA (0.842) and between PBB and height (-0.578) show that selection based on PBB will lead to height reduction. The type of selection proved itself efficient for increasing rubber yield while substantially reducing plant height and less reducing trunk perimeter. All these effects are desirable in a rubber tree genetic improvement program aiming at selection of genotypes suitable for higher density plantations.

Key words: Hevea brasiliensis, genetic improvement, rubber yield, short plant height.

\section{INTRODUÇÃO}

A seringueira [Hevea brasiliensis (Willd ex Adr. de Juss.) Muell.-Arg.] é cultivada no Brasil, principalmente nas denominadas áreas de escape do mal-das-folhas que se localizam nos seguintes Estados: São Paulo, Mato Grosso, Mato Grosso do Sul, Bahia e no noroeste do Paraná (Pereira, 1992). Recentemente, a espécie tem expandido suas áreas de plantio nesses Estados e esse fato justifica a realização de programas de melhoramento da espécie nesses locais.

Diversos trabalhos que visam maximizar os ganhos genéticos da seringueira foram realizados no Sri Lanka (JAYASEKERA, 1983), Indonésia (DASLIN et al., 1986), Nigéria (OKNOPISE et al., 1986), Índia (MEENATTOR et al., 1991), Malásia (TAN, 1995) e Brasil (GoNÇALVES et al., 1998; Costa et al., 2000b; Costa et al., 2002; GONÇALVES et al., 2005; FURLANI et al., 2005), dentre outros.

Os testes de progênies, instrumentos importantes para o trabalho do melhorista, são usados na estimativa de parâmetros genéticos e seleção de indivíduos, quando se procura avaliar a magnitude e a natureza da variância genética disponível, com vistas a quantificar e maximizar os ganhos genéticos, utilizando-se procedimento de seleção adequado. Os caracteres normalmente avaliados nesses experimentos com a seringueira referem-se à altura, perímetro do caule e produção de látex, sendo a seleção geralmente baseada nesse último.

Simmonds (1989) resume os principais caracteres objetivos do melhoramento da seringueira como alta produção de látex (com ênfase em sangria e produtividade precoces), resistência ao vento e a doenças foliares. A produção de látex é determinada pela taxa de partição de foto-assimilados em látex e pela capacidade de retenção do vigor e crescimento sob sangria. SimMONDS (1989) relata também, que em plantas mais baixas e com internódios mais curtos observaram, também, as seguintes vantagens: permitem maior densidade de plantio; potencialmente, aumentam a partição de foto-assimilados em látex em detrimento do crescimento em altura; há menores danos ocasionados pelo vento bem como maior facilidade na colheita.
No entanto, o efeito da seleção simultânea para porte baixo e alta produção de látex não foi ainda adequadamente estudado em seringueira. Com base nesses antecedentes, os objetivos deste trabalho foram avaliar a variabilidade genética e a possibilidade de sucesso na seleção simultânea para porte baixo e alta produção de látex, com novas variáveis relacionais: a produtividade de látex por área basal e a produtividade de látex por unidade de altura das plantas. A seleção baseada nessas novas variáveis podem permitir a identificação de materiais genéticos mais produtivos destinados a plantios adensados.

\section{MATERIAL E MÉTODOS}

O ensaio foi instalado na Estação Experimental de Votuporanga (SP), pertencente ao Instituto Agronômico (IAC) de Campinas, localizada na latitude $20^{\circ} 25^{\prime} \mathrm{S}$, longitude $49^{\circ} 59^{\prime} \mathrm{W}$ e altitude de 450 $\mathrm{m}$. O solo é do tipo Latossolo Vermelho-Escuro, de textura média, em clima tropical continental, com predominância de verão úmido e um período de inverno seco com temperaturas e precipitações pluviais mais reduzidas. A temperatura média anual é de $20^{\circ} \mathrm{C}$ e pluviosidade média anual é de $1420 \mathrm{~mm}$.

O material genético que compõe o estudo é constituído de 22 progênies de meios-irmãos provenientes de sementes de polinização aberta, obtidas de 22 clones parentais selecionados fenotipicamente em vista da maior produção de látex em uma população-base de Hevea brasiliensis, de origem asiática.

As sementes foram colocadas para germinar em sacos de polietileno, e as progênies transportadas para o local definitivo, com dois lançamentos foliares. As progênies foram instaladas sob o delineamento de blocos ao acaso com 22 tratamentos, cinco repetições e dez plantas por parcela, no espaçamento de $3 \times 2 \mathrm{~m}$ em linhas simples.

Aos três anos de idade, as progênies foram avaliadas quanto aos caracteres: altura total (m); perímetro na altura da base do caule $(\mathrm{cm})$; produção 
de borracha seca $(\mathrm{g})$, obtida pelo teste Hamaker-MorrisMann (HMM), modificado para plantas de três anos de idade (TAN e Subramanian, 1976), utilizando-se a média de produção de borracha seca de 30 cortes por planta. O painel de sangria foi aberto a $20 \mathrm{~cm}$ do solo, pelo sistema $\mathrm{S} / 2 \mathrm{~d} / 3$, no total de 35 cortes, descartando-se as cinco primeiras amostras que correspondem à fase de amansamento do painel. A nomenclatura S/ 2 corresponde ao corte em meia espiral, e a nomenclatura d/ 3 expressa o intervalo entre sangrias, ou seja, uma sangria a cada três dias. A partir desses caracteres, foram criadas as variáveis relacionais PBA - produção de borracha por unidade de altura, calculada através da razão entre os valores de produção de borracha seca $(\mathrm{g} / \mathrm{m})$ por $\mathrm{m}$, proveniente dos valores de altura total das progênies e, PBB produção de borracha por unidade de área basal da planta, obtida por meio da razão entre os valores de produção de borracha seca $\left(\mathrm{g} \mathrm{cm}^{-2}\right)$, proveniente dos valores do perímetro do caule das progênies.

As variáveis foram analisadas usando-se o método de modelo linear misto via procedimento REML/BLUP (máxima verossimilhança restrita / melhor preditor linear não viciado) apresentado por Resende (2002a), por meio do seguinte modelo estatístico:

$$
y=X b+Z a+W c+e,
$$

em que: $y, b, a, c$ e e: vetores de dados, dos efeitos de blocos (fixos), de efeitos genéticos aditivos (aleatório), de efeitos de parcela (aleatório) e de erros aleatórios respectivamente.

$\mathrm{X}, \mathrm{Z}$ e W: matrizes de incidência para b, a e c respectivamente.

A partir dos componentes de variância associados aos referidos efeitos, foram estimados os seguintes parâmetros:

$$
\hat{h}_{a}^{2}=\frac{\hat{\sigma}_{a}^{2}}{\hat{\sigma}_{a}^{2}+\hat{\sigma}_{c}^{2}+\hat{\sigma}_{e}^{2}}=\text { herdabilidade individual no }
$$

sentido restrito;

$$
\hat{c}^{2}=\hat{\sigma}_{c}^{2} /\left(\hat{\sigma}_{a}^{2}+\hat{\sigma}_{c}^{2}+\hat{\sigma}_{e}^{2}\right)=\text { correlação devida ao }
$$
ambiente comum da parcela;

$$
\hat{h}_{m p}{ }^{2}=\frac{0,25 \hat{\sigma}_{a}^{2}}{0,25 \hat{\sigma}_{a}^{2}+\hat{\sigma}_{c}^{2} / b+\hat{\sigma}_{e}^{2} /(n b)}=\text { herdabilidade }
$$

média de progênies;

$$
\begin{aligned}
& \hat{r}_{\hat{a} a}=\sqrt{\hat{h}_{m p}^{2}}=\text { acurácia seletiva; } \\
& \hat{\sigma}_{f}^{2}=\hat{\sigma}_{a}^{2}+\hat{\sigma}_{d}^{2}+\hat{\sigma}_{e}^{2}=\text { variância fenotípica; } \\
& \mathrm{CV}_{\mathrm{gi}}(\%)=\frac{\sqrt{\hat{\sigma}_{a}^{2}}}{\bar{X}} \cdot 100=\text { coeficiente de variação }
\end{aligned}
$$
genética individual;

$\mathrm{CV}_{\mathrm{e}}(\%)=\frac{\sqrt{\hat{\sigma}_{e}^{2}}}{\bar{x}} \cdot 100=$ coeficiente de variação ambiental dentro dă parcela.

em que: $\hat{\sigma}_{a}^{2}=$ variância genética aditiva;

$\hat{\sigma}_{\text {parc }}^{2}=$ variância entre parcelas;

$\hat{\sigma}_{e}^{2}=$ variância residual dentro de parcelas (ambiental + não aditiva);

$$
\hat{\sigma}_{d}^{2}=\text { variância genética de dominância. }
$$

As estimativas dos parâmetros genéticos foram obtidas pelo procedimento SELEGEN REML/ BLUP a partir de iterações nas equações de modelo misto (RESENDE, 2002b).

\section{RESULTADOS E DISCUSSÃO}

Os resultados referentes às estimativas dos parâmetros genéticos para os caracteres altura (A), perímetro do caule $(\mathrm{C})$, produção de borracha seca (PB), produção de borracha por unidade de altura (PBA) e produção de borracha por unidade de área basal da planta (PBB) estão relacionados na tabela 1.

Os coeficientes de variação genética individual $\left(\mathrm{CV}_{\mathrm{gi}} \%\right)$, que expressam em porcentagem da média geral a quantidade de variação genética existente, revelaram valores moderados para os caracteres altura e perímetro $(23,32 \%$ e $17,08 \%)$, e elevados para os demais caracteres que envolvem a produção de borracha seca, por unidade de altura e de área basal, respectivamente de $44,33 \%$ e $89,46 \%$. Estes resultados são condizentes àqueles obtidos por MORETI et al. (1994), Bоock et al. (1995) e Costa et al. (2000b) revelando que a população pode ser considerada apropriada para o programa de melhoramento genético. Em outros termos, ganho genético é esperado aplicando-se procedimento adequado de seleção.

Os coeficientes de variação residual $\left(\mathrm{CV}_{\mathrm{e}} \%\right)$ resultaram em valores baixos para altura e perímetro, e altos para os caracteres envolvendo a produção de borracha, conforme GARCIA et al. (1989). No entanto, nos caracteres envolvendo a produção de borracha ocorreram também altos coeficientes de variação genética, fato que conduziu a altos coeficientes de herdabilidade em nível de médias de progênies.

As estimativas dos coeficientes de herdabilidades individuais variaram de $26 \%$ a $84 \%$, portanto, de magnitudes moderadas a altas. Esses resultados são condizentes com aqueles observados por Paiva et al. (1982), Alika (1985) e Costa et al. (2000a) para a seringueira. Ressaltam-se os valores mais altos das herdabilidades de médias de progênies $(77 \%$ a $89 \%)$, cuja seleção pode ser mais efetiva utilizando-se as informações das famílias. Tais resultados são coerentes com os observados na literatura relativa à seringueira (GILBERT et al., 1973; Nga e Subramanian, 1974; Moreti et al., 1994, Costa et al., 2000b e GonçALVES et al., 2005). 
Tabela 1. Estimativas de parâmetros genéticos para os caracteres altura (m), perímetro (cm), produção de borracha (g), produção de borracha por unidade de altura $(\mathrm{g} / \mathrm{m})$ e produção de borracha por unidade de área basal da planta $\left(\mathrm{g} \mathrm{cm}^{2}\right)$, média geral e erro-padrão da seringueira, aos três anos de idade, em Votuporanga (SP)

\begin{tabular}{lccccc}
\hline Estimativas $\left(^{1}\right)$ & Altura & Perímetro & $\begin{array}{c}\text { Produção de } \\
\text { borracha seca }\end{array}$ & $\begin{array}{c}\text { Produção de borracha } \\
\text { por unidade de altura }\end{array}$ & $\begin{array}{c}\text { Produção por unidade } \\
\text { de área basal }\end{array}$ \\
\hline $\mathrm{m}$ & $\mathrm{cm}$ & $\mathrm{g}$ & $\mathrm{g} / \mathrm{m}$ & $\mathrm{g} / \mathrm{cm}^{2}$ & 0,6187 \\
$\hat{h}_{a}^{2}$ & 0,8396 & 0,5408 & 0,2610 & 0,4060 & 0,8852 \\
$\hat{h}_{m p}^{2}$ & 0,8816 & 0,8728 & 0,7708 & 0,8438 & 0,9408 \\
$\hat{r}_{\hat{a} a}$ & 0,9389 & 0,9343 & 0,8780 & 0,9186 & $1,39.10-4$ \\
$\hat{\sigma}_{a}^{2}$ & 0,5137 & 20,6490 & 0,0847 & 0,0167 & $4.10-6$ \\
$\hat{\sigma}_{\text {parc }}^{2}$ & 0,0420 & 0,5102 & 0,0012 & 0,0002 & $8,2.10-5$ \\
$\hat{\sigma}_{e}^{2}$ & 0,0560 & 17,0225 & 0,2387 & 0,0243 & $2,24.10-4$ \\
$\hat{\sigma}_{f}^{2}$ & 0,6118 & 38,1818 & 0,3247 & 0,0412 & $0,01317 \pm 0,0002$ \\
\hline Média geral & $3,0732 \pm 0,0160$ & $26,6020 \pm 0,1058$ & $0,6568 \pm 0,0097$ & $0,2232 \pm 0,0039$ & 89,46 \\
\hline CVgi (\%) & 23,32 & 17,08 & 44,33 & 58,02 & 36,01 \\
CVe (\%) & 9,55 & 7,29 & 27,02 & 27,90 & 0 \\
\hline
\end{tabular}

(1) Herdabilidade individual no sentido restrito $\left(\hat{h}_{a}^{2}\right)$, herdabilidade média de progênie $\left(\hat{h}_{m p}^{2}\right)$, acurária seletiva $\left(\hat{r}_{\hat{a} a}\right)$, variância genética aditiva $\left(\hat{\sigma}_{a}^{2}\right)$, variância ambiental entre parcelas $\left(\hat{\sigma}_{\text {parc }}^{2}\right)$, variância residual dentro de parcela (ambiental + não aditiva, $\left.\hat{\sigma}_{e}^{2}\right)$, variância fenotípica individual $\left(\hat{\sigma}_{f}^{2}\right)$, coeficiente de variação genética individual (CVgi \%), coeficiente de variação ambiental dentro de parcelas (CVe\%).

A mais importante função da herdabilidade no estudo genético do caráter métrico é seu papel preditivo expressando a confiança do valor fenotípico como um guia para o valor genético, ou o grau de correspondência entre o valor fenotípico e o valor genético (FALCONER, 1987; Vencovsky e BARRIGA, 1992). Segundo Falconer (1987), a herdabilidade é uma propriedade não somente de um caráter, mas também da população e das circunstâncias de ambientes às quais os indivíduos estão sujeitos. O valor da herdabilidade poderá ser afetado se houver alteração em qualquer um dos componentes da variância.

As herdabilidades individuais para os novos caracteres PBA e PBB foram de magnitudes mais elevadas (41\% e $62 \%$ ) do que para o caráter produção de borracha (26\%). Esse fato revela que a combinação de dois caracteres em novo caráter aumentou a variabilidade genética disponível para seleção e, também, que é possível realizar eficiente melhoramento genético simultâneo para o aumento da produção de borracha e redução do porte da planta.

A acurácia ou correlação entre os valores genéticos preditos e os verdadeiros variou de $88 \%$ a 94\% (Tabela 1). Segundo RESENDE (2002), a acurácia é uma medida que está associada à precisão na seleção, sendo o principal componente do progresso genético que o melhorista pode alterar, visando maximizar o ganho genético. A acurácia pode ser incrementada por meio de uma experimentação mais adequada, mantendo-se o mesmo tamanho do experimento, porém alterando-se o número de indivíduos por parcela e de repetições (RESENDE, 2002). As acurácias verificadas neste trabalho são condizentes com aquelas constatadas por Costa et al. (2000a) para o caráter produção de borracha seca em seringueira.

Na tabela 2, constam as correlações genéticas, envolvendo os cinco caracteres avaliados.

Tabela 2. Correlações genéticas, envolvendo os cinco caracteres avaliados em progênies de seringueira, aos três anos de idade, no município de Votuporanga (SP)

\begin{tabular}{lccccc}
\hline Variáveis & Altura & Perímetro & PB & PBA & PBB \\
\hline Altura & 1 & $0,72^{*}$ & $-0,08 \mathrm{~ns}$ & $-0,48^{* *}$ & $-0,58^{*}$ \\
Perímetro & - & 1 & $0,27^{* *}$ & $-0,10 \mathrm{~ns}$ & $-0,50^{* *}$ \\
PB & - & - & 1 & $0,90^{*}$ & $0,63^{*}$ \\
PBA & - & - & - & 1 & $0,84^{*}$ \\
PBB & - & - & - & - & 1 \\
\hline
\end{tabular}

* $(\mathrm{p}<0,01) ; * *(\mathrm{p}<0,05)$, ns : não significativo, PB : Produção de borracha seca, PBA : Produção de borracha por unidade de altura, PBB : Produção por unidade de área basal.

Verifica-se que as correlações genéticas dos novos caracteres (PBA e PBB) com a produção de borracha é alta, mas não tendendo a 1, especialmente a correlação entre $\operatorname{PB}$ e $\operatorname{PBB}(0,631)$. Evidencia-se que é possível melhorar os dois caracteres (PB e PBB) simultaneamente, não havendo prejuízo no caráter principal (PB). Isto é explicado pelas baixas correlações genéticas entre os caracteres PB e altura e PB e perímetro (Tabela 
2). Adicionalmente, as altas correlações entre PBB e PBA $(0,841)$ e entre $\mathrm{PBB}$ e altura $(-0,58)$ revelam que a seleção baseada em $\mathrm{PBB}$ resultará em redução da altura. Essa seleção, logicamente, conduzirá também a plantas com maior produção por unidade de área basal, fato que pode possibilitar a adoção de plantios mais adensados. Assim, a seleção realizada terá como base a variável PBB.

$\mathrm{Na}$ tabela 3, estão indicados os valores genéticos das cinco melhores progênies para PBB, bem como os seus valores genéticos para os demais caracteres. São apresentados também o ganho genético direto com a seleção para PBB e os ganhos indiretos nos demais caracteres, com seleção das cinco melhores progênies para PBB.

Constata-se que com a seleção das cinco melhores progênies para $\mathrm{PBB}$, obtém-se ganhos genéticos positivos de 56,6\% em PBB, 16,2\% em PB, $30 \%$ em PBA e ganhos genéticos negativos de 10,3\% em altura e 5,5\% em perímetro. Assim, esse tipo de seleção foi eficiente em aumentar a produção de borracha, reduzir bastante a altura e pouco o perímetro das plantas. Todos esses efeitos são desejáveis em um programa de melhoramento da seringueira, visando à seleção de genótipos para plantios adensados.

Tabela 3. Valores genéticos (VG) para produção de borracha por unidade de área basal da planta (PBB), bem como os seus valores genéticos para os demais caracteres e, ganho genético direto com a seleção para PBB e os ganhos indiretos nos demais caracteres, com seleção das 5 melhores progênies para PBB, aos três anos de idade, em Votuporanga (SP)

\begin{tabular}{|c|c|c|c|c|c|}
\hline \multirow{2}{*}{ Progênies } & \multicolumn{5}{|c|}{ Valores genéticos (VG) } \\
\hline & PBB & Altura & Perímetro & $\mathrm{PB}$ & PBA \\
\hline 13 & 0,0317 & 2,6382 & 24,3410 & 0,8963 & 29,87 \\
\hline 22 & 0,0208 & 2,4180 & 21,6083 & 0,5564 & 23,84 \\
\hline 4 & 0,0189 & 2,5576 & 23,2893 & 0,7060 & 21,73 \\
\hline 17 & 0,0172 & 3,2435 & 28,6075 & 0,8694 & 20,65 \\
\hline 6 & 0,0145 & 2,9191 & 27,8931 & 0,7889 & 19,98 \\
\hline Ganho Genético (\%) & 56,6 & $-10,3$ & $-5,5$ & 16,2 & 30,9 \\
\hline
\end{tabular}

PB : Produção de borracha seca, PBA : Produção de borracha por unidade de altura, PBB : Produção por unidade de área basal.

\section{CONCLUSÕES}

1. A combinação dos caracteres produção de borracha $x$ altura e produção de borracha x perímetro aumentou a variabilidade genética disponível para seleção e pode propiciar um eficiente melhoramento genético simultâneo para o aumento da produção de borracha e redução do porte da planta.

2. As correlações genéticas dos novos caracteres (PBA e PBB) com a produção de borracha indicam que existe possibilidade de melhorar os dois caracteres (PB e PBB), ao mesmo tempo, não havendo prejuízo no caráter principal $(\mathrm{PB})$. As altas correlações entre PBB e PBA $(0,841)$ e entre PBB e altura $(-0,58)$ evidenciam que a seleção baseada em PBB resultará em redução da altura.

3. A seleção simultânea foi eficiente em aumentar a produção de borracha, reduzindo a altura e ligeiramente a circunferência das plantas.

\section{AGRADECIMENTOS}

Os autores agradecem às Instituições CNPq e CAPES pelas bolsas concedidas.

\section{REFERÊNCIAS}

ALIKA, J.E. Heritability and genotypic gain from selection rubber (Hevea brasiliensis). Silvae Genetica, Frankfurt, v.34, n.1, p.1-4, 1985.

BOOCK, M.V.; GONÇALVES, P.S.; BORTOLETTO, N.; MARTINS, A.L.M. Herdabilidade, variabilidade genética e ganhos genéticos para produção e caracteres morfológicos em progênies jovens de seringueira. Pesquisa Agropecuária Brasileira, Brasília, v.30, n.5, p.673-681, 1995.

COSTA, R.B.; RESENDE, M.D.V.; ARAUJO, A.J.; GONÇALVES, P.S.; BOTOLETTO, N. Seleção combinada univariada e multivariada aplicada ao melhoramento genético da seringueira. Pesquisa Agropecuária Brasileira, Brasília, v.35, n.2, p. 381-388, 2000a.

COSTA, R.B.; RESENDE, M.D.V.; GONÇALVES, P. de S. Selection and genetic gain in populations of Hevea brasiliensis with a mixed mating system. Genetics and Molecular Biology, Ribeirão Preto, v.23, n.3, p.671-679, 2000b.

COSTA, R.B.; RESENDE, M.D.V.; GONÇALVES, P. de S.;SILVA, M.A. Individual multivariate REML/BLUP in the presence of genotype $x$ environment interaction in rubber tree (Hevea) breeding. Crop breeding and Applied Biotechnology, Viçosa, v.2, n.1, p.131-140, 2002. 
DASLIN, A.; BAIHAKI, A.; DANAKUSUMA, T.M.; HAERUMAN, M.S. Genotypes $x$ environment interaction in rubber and their implications in clonal selection. Bull. Perkaretan, n.4, p.23-28, 1986.

FALCONER, D. S. Introdução à genética quantitativa. Viçosa: Universidade Federal de Viçosa, 1987. 279p.

FURLANI, R. C. M.; MORAES, M. L. T.; RESENDE, M. D. V.; FURLANI JUNIOR, E.; GONÇALVES, P.S.; VALÉRIO FILHO, W. V.; PAIVA, J. R. Estimation of variance components and prediction of breeding values in rubber tree breeding using the REML/BLUP procedure. Genetics and Molecular Biology, Ribeirão Preto, v. 28, n. 2, p. 271-276, 2005.

GARCIA, C.H. Tabela para classificação do coeficiente de variação. Piracicaba: Instituto de Pesquisas e Estudos Florestais-IPEF. 1989. (Circular Técnica 171)

GILBERT, N.E.; DODDS, K.S.; SUBRAMANIAN, S. Progress of breeding investigations with Hevea brasiliensis. Analysis of data from earlier crosses. Journal of the Rubber Research Institute of Malaysia, Kuala Lumpur, v.3, n.5, p.365-380, 1973.

GONÇALVES, P.S.; BATAGLIA, O.C.; SANTOS, W.R.; ORTOLANI, A.A.; SEGNINI JUNIOR, I.; SHIKASHO, E.H. Growth trends, genotype-environment interaction and genetic gains in six-years old rubber tree clones (Hevea) in São Paulo State, Brazil. Genetics and Molecular Biology, Ribeirão Preto, v.21, n.1, p.115-122, 1998.

GONÇALVES, P.S.; MORAES, M.L.T.; BOROLETTO, N.; COSTA, R.B.; GONÇALVES, E.C.P. Genetic variation in growth traits and yield of rubber trees (Hevea brasiliensis) growing in the Brazilian state of São Paulo. Genetics and Molecular Biology, Ribeirão Preto, v.28, n.4, p.551-558, 2005.

JAYASEKERA, N.E.M. A basis for selecting Hevea clones stable to unpredictable agroclimatic variability. Silvae Genetica, Frankfurt, n.32, p.181-185, 1983.

MEENATTOR, R.J.; VINOD K.K.; KRUSHNAKUMAR, A.K.; SETURAJ, M.R.; POTTY, S.N.; SINHÁ, R.R. Clone $x$ environment interaction during early growth phase of Hevea brasiliensis clonal stability on diameter. Journal of the Rubber Research Institute of Malaysia, Kuala Lumpur, n.4, p.51-58, 1991.

MORETI, D.; GONÇALVES, P.S.; GORGULHO, E.P.; MARTINS, A.L.M.; BORTOLETTO, N. Estimativas de parâmetros genéticos e ganhos esperados com a seleção de caracteres juvenis em progênies de seringueira. Pesquisa Agropecuária Brasileira, Brasília, v.29, n.7, p.1099-1109, 1994.

NGA, B.H.; SUBRAMANIAN, S. Variation in Hevea brasiliensis. I. Yield and girth data of the 1937 hand pollinated seedlings. Journal of the Rubber Research Institute of Malaysia, Kuala Lumpur, v. 24, n.2, p.69-74, 1974.

OKNOPISE, O.U.; OLAPADA, O.; MEKAKO, H.U. Genotype X environment interaction in Hevea brasiliensis. Journal of the Rubber Research Institute of Malaysia, Kuala Lumpur, n.46, p.506-511, 1986.
PAIVA, J.R.; MIRANDA FILHO, J.B.;SIQUEIRA, E.R.; VALOIS, A.C.C. Predição do ganho de alguns caracteres em seringueira em três esquemas de seleção. Pesquisa Agropecuária Brasileira, Brasília, v.17, n.11, p.1646-1653, 1982.

PEREIRA, J.P. Seringueira: formação de mudas, manejo e perspectivas no noroeste do Paraná. Londrina: IAPAR, 1992. 60p. (IAPAR. Circular Técnica, 700)

RESENDE, M.D.V. Genética biométrica e estatística no melhoramento de plantas perenes. Brasília: Embrapa Informação Tecnológica. 2002a. 975p.

RESENDE, M.D.V. Software Selegen Reml/Blup. Colombo: Embrapa Florestas, 2002b. 67p. (Documentos, 77)

SIMMONDS, N.W. Rubber breeding. In: WEBSTER, C.C.; BAULKWILL, W,J. (Eds.). Rubber. London: Longman, 1989. p. 85-124.

TAN, H.; SUBRAMANIAN, S.A. A five-parent diallel cross analysis for certain characters of young Hevea seedlings. In: INTERNACIONAL RUBBER CONFERENCE, Kuala Lumpur, 1975. Proceedings... v. 2, p. 13-16, 1976.

TAN, H. Genotype x environment interaction studies In: rubber (Hevea) clones. Journal of the Rubber Research Institute of Malaysia, Kuala Lumpur, n. 10, p. 63-76, 1995.

VENCOVSKY, R.; BARRIGA, P. Genética biométrica no fitomelhoramento. Ribeirão Preto: Sociedade Brasileira de Genética, 1992. 496p. 